\title{
ERRATUM
}

Jin-Yeon Kim • Jun-Shin Lee

\section{Erratum to: Extinction cross-section for elastic wave scattering in energy-absorbing media: revisited}

Received: 25 October 2009 / Revised: 16 January 2010 / Published online: 4 March 2010

(C) Springer-Verlag 2010

\section{Erratum to: Acta Mech (2009) 207:153-161}

\section{DOI 10.1007/s00707-008-0113-8}

Errors in the asymptotic evaluation of integrals in the original manuscript [1] are corrected here. The extinction power detected on the detector area $A_{D}$ is obtained as

$$
\begin{aligned}
P^{\mathrm{ext}}= & \omega \operatorname{Im}\left[i(\lambda+2 \mu) k_{p} \operatorname{Re} \int_{A_{D}} \hat{r}_{1} A_{k}^{(p)} a_{k}^{(p)} h_{p}(r) \mathrm{e}^{i k_{p}^{(1)}(r-x)-k_{p}^{(2)}(r+x)} \mathrm{d} S\right. \\
& \left.+i \mu k_{s} \operatorname{Re} \int_{A_{D}} \hat{r}_{1} A_{k}^{(s)} a_{k}^{(s)} h_{s}(r) \mathrm{e}^{i k_{s}^{(1)}(r-x)-k_{s}^{(2)}(r+x)} \mathrm{d} S\right] .
\end{aligned}
$$

This is Eq. (23) in the original manuscript but in a corrected form. Note that since the polarization vectors $a_{k}^{(p)}$ and $a_{k}^{(s)}$ are real vectors, the conjugate signs (overbars) on them are removed. The integrals in Eq. (1) are in the following form:

$$
J=\int_{A_{D}} \eta(y, z) \mathrm{e}^{i k x \xi(y, z)} \mathrm{d} S .
$$

The asymptotic value of this integral can be obtained by applying the method of stationary phase [2,3]. The only meaningful stationary point is located in the forward direction for both two- and three-dimensional configurations $\left(z_{s}=0\right.$ for the two-dimensional case and $y_{s}=0, z_{s}=0$, for the three-dimensional case, respectively). Then, the leading term of the integral Eq. (2) is

$$
J \sim \frac{2 \pi i x}{k} \eta(0,0)
$$

The online version of the original article can be found under doi:10.1007/s00707-008-0113-8.

J.-Y. Kim $(\varangle)$

School of Civil and Environmental Engineering, Georgia Institute of Technology,

Atlanta, GA 30322, USA

E-mail: jk290@mail.gatech.edu; jykim.ADA@gmail.com; jinyeon.kim@me.gatech.edu

J.-S. Lee

Nuclear Power Research Laboratory, Korea Electric Power Company,

Taejon 305-380, South Korea 
Evaluation of the integrals in Eq. (1) yields

$$
P^{\mathrm{ext}}=\frac{\omega}{2} \operatorname{Im}\left[i(\lambda+2 \mu) k_{p} \mathrm{e}^{-2 k_{p}^{(2)} x}\right] \cdot \frac{4 \pi}{k_{p}^{(1)}} \operatorname{Im}\left[A_{k}^{(p)}(0) a_{k}^{(p)}\right]+\frac{\omega}{2} \operatorname{Im}\left[i \mu k_{s} \mathrm{e}^{-2 k_{s}^{(2)} x}\right] \cdot \frac{4 \pi}{k_{s}^{(1)}} \operatorname{Im}\left[A_{k}^{(s)}(0) a_{k}^{(s)}\right] .
$$

A mistake in the old expressions in [1] is that Eq. (23) contains the complex wavenumbers $k_{p}$ and $k_{s}$ instead of the real wavenumbers $k_{s}^{(1)}$ and $k_{s}^{(1)}$, which are thus out of the Im operators in this equation.

The intensity of the incident wave is given by

$$
I_{1}^{\mathrm{inc}}=\frac{\omega}{2} \operatorname{Im}\left[i(\lambda+2 \mu) k_{p}\left|a_{k}^{(p)}\right|^{2} \mathrm{e}^{-2 k_{p}^{(2)} x}+i \mu k_{s}\left|a_{k}^{(s)}\right|^{2} \mathrm{e}^{-2 k_{s}^{(2)} x}\right] .
$$

From Eqs. (4) and (5) and the definition of the extinction cross-section [4,5], the extinction cross-sections are

$$
\Sigma^{\mathrm{ext}}=-\frac{4 \pi}{k_{p}^{(1)}\left|a_{k}^{(p)}\right|^{2}} \operatorname{Im}\left[A_{k}^{(p)}(0) a_{k}^{(p)}\right]
$$

for longitudinal wave incidence, and

$$
\Sigma^{\mathrm{ext}}=-\frac{4 \pi}{k_{s}^{(1)}\left|a_{k}^{(s)}\right|^{2}} \operatorname{Im}\left[A_{k}^{(s)}(0) a_{k}^{(s)}\right]
$$

for shear wave incidence.

A similar derivation for the two-dimensional case yields the extinction power at the detector

$$
P^{\mathrm{ext}}=\frac{\omega}{2} \operatorname{Im}\left[i(\lambda+2 \mu) k_{p} \mathrm{e}^{-2 k_{p}^{(2)} x}\right] \cdot \frac{4}{k_{p}^{(1)}} \operatorname{Re}\left[A_{k}^{(p)}(0) a_{k}^{(p)}\right]+\frac{\omega}{2} \operatorname{Im}\left[i \mu k_{s} \mathrm{e}^{-2 k_{s}^{(2)} x}\right] \cdot \frac{4}{k_{s}^{(1)}} \operatorname{Re}\left[A_{k}^{(s)}(0) a_{k}^{(s)}\right],
$$

and the extinction cross-sections are

$$
\Sigma^{\mathrm{ext}}=-\frac{4}{k_{p}^{(1)}\left|a_{k}^{(p)}\right|^{2}} \operatorname{Re}\left[A_{k}^{(p)}(0) a_{k}^{(p)}\right]
$$

for longitudinal wave incidence, and

$$
\Sigma^{\mathrm{ext}}=-\frac{4}{k_{s}^{(1)}\left|a_{k}^{(s)}\right|^{2}} \operatorname{Re}\left[A_{k}^{(s)}(0) a_{k}^{(s)}\right]
$$

for in-plane vertically polarized incidence (SV wave scattering). The extinction cross-section for horizontally polarized (in the "3" direction) shear wave scattering (SH wave scattering) can also be derived in a similar manner,

$$
\Sigma^{\mathrm{ext}}=-\frac{4}{k_{s}^{(1)} a_{3}^{(s)}} \operatorname{Re}[C(0)],
$$

where $C(0)$ is the amplitude of the scattered $\mathrm{SH}$ wave amplitude in the forward direction.

Finally, note that these expressions are in the same form as the extinction cross-section for electromagnetic wave scattering in an absorbing medium [6].

Acknowledgments The author thanks Michael I. Mishchenko of NASA Goddard Institute for Space Studies for bringing the present issue and Ref. [6] to his attention. 


\section{References}

1. Kim, J.-Y., Lee, J.-S.: Extinction cross-section for elastic wave scattering in energy-absorbing media: revisited. Acta Mech. 207, 153-161 (2009)

2. Born, M., Wolf, E.: Principles of Optics, 6th edn. Pergamon, Oxford (1987)

3. Erdelyi, A.: Asymptotic Expansions. Dover, New York (1965)

4. Kim, J.-Y.: Extinction and propagation of elastic waves in inhomogeneous. Mech. Mater. 35, 877-884 (2003)

5. Kim, J.-Y.: Extinction of elastic wave energy due to scattering in a viscoelastic medium. Int. J. Solids Struct. 40, 4319$4329(2003)$

6. Mishchenko, M.I.: Electromagnetic scattering by a fixed finite object embedded in an absorbing medium. Opt. Express 15, 13188-13202 (2007) 